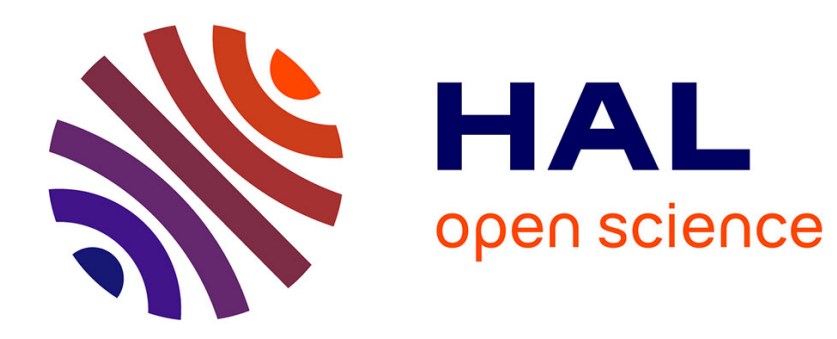

\title{
Can a metal-insulator transition induce s-wave superconductivity?
}

Philippe Nozières

\section{To cite this version:}

Philippe Nozières. Can a metal-insulator transition induce s-wave superconductivity?. 2004. hal00001405

\section{HAL Id: hal-00001405 \\ https://hal.science/hal-00001405}

Preprint submitted on 3 Apr 2004

HAL is a multi-disciplinary open access archive for the deposit and dissemination of scientific research documents, whether they are published or not. The documents may come from teaching and research institutions in France or abroad, or from public or private research centers.
L'archive ouverte pluridisciplinaire HAL, est destinée au dépôt et à la diffusion de documents scientifiques de niveau recherche, publiés ou non, émanant des établissements d'enseignement et de recherche français ou étrangers, des laboratoires publics ou privés. 


\title{
Can a metal-insulator transition induce s-wave superconductivity?
}

\author{
Ph. Nozières \\ LEPES, Centre National de la Recherche Scientifique \\ BP166, 38042 Grenoble Cedex9
}

November 282002

\begin{abstract}
A recent paper of Capone et.al. has studied an extended Hubbard model, in which local orbital degrees of freedom allow an even integer occupation at each site.A strong local repulsion $U$ triggers a metal-insulator transition. Within a DMFT numerical analysis they show that when the ground state is a singlet a pocket of s-wave superconductivity appears in the vicinity of the Mott transition, with a strongly enhanced superconducting gap. A qualitative understanding of their result is proposed, and suggestions are made of possible systems in which this beautiful effect might be searched.
\end{abstract}

\section{Introduction}

It has been known for many years that a lattice gas with an integer filling $N=p N_{L}$ (where $p$ is an integer and $N_{L}$ the number of lattice sites) will turn into an insulator if the local electron repulsion $U$ is strong enough. Then the primary feature of the level structure is the valency $p$ : charge excitations towards states with a valency $p \pm 1$ face an energy gap, hence the insulating behaviour. Degeneracies within a given valence state do not affect such a metal-insulator transition: for large $U$ they remain minor perturbations as well as the kinetic energy due to electron hopping. Of course all of that breaks down in doped systems, when $p$ is not an integer: the system is always a conductor - albeit a strange one close to the Mott transition. 
The standard example is the usual Hubbard model with no orbital degree of freedom: spin $1 / 2$ electrons hop with a band width $D$. The only non trivial integer valency is $p=1$ : in the insulating state each site is a spin doublet. These localized spins are coupled via Anderson superexchange: they usually order at low temperatures. Antiferromagnetism hides the pure Mott transition which is never reached. If frustration is large enough one might envisage instead an incoherent paramagnetic insulator retaining a residual spin entropy $S_{o}=N_{L} \ln 2$ at zero temperature. Then the Mott transition may be viewed as a Kondo alloy problem: free electrons appear below a critical $U_{c}$ and they quench that entropy , turning the paramagnetic insulator into a regular paramagnetic Fermi liquid. As a result the transition is first order, conductor and insulator coexisting in a finite range $U_{c 1}<U_{c 2}$. That problem has been extensively discussed in the limit of infinite dimension, using "dynamical mean field theory" (DMFT) [1].

The problem is much richer if electrons have orbital degeneracy $g$. Then there are $2 g$ one electron states per site and a non trivial Mott insulator may exist for any valency $p$ between 1 and $(2 g-1)$. The degeneracy between the $C_{2 g}^{p}$ atomic states with valency $p$ is lifted by local interactions, crystal field and spin-orbit coupling. According to Hund's rule the ground state should be the one with maximum multiplicity : let us explore the inverse situation in which the ground state has minimum degeneracy. For an even valency that will be a singlet, both orbital and spin. One may argue that fighting against Hund's rule is unreasonable (it is based on exchange!). We take it as a model: there are other ways to achieve a singlet ground state, as we shall see later. In such a case the Mott insulator has no residual entropy at $T=0$, and the Mott transition is not masked by translational symmetry breaking such as antiferromagnetism. The interaction $J$ that brings the singlet down acts as an attraction of two electrons in the $s$ wave channel: could such a trend induce superconductivity? One may formulate the question in a simple specific example, namely $L=1$ electrons and a valency $p=2$ : can the Mott transition create superconductivity?

That question has been studied recently in a beautiful paper by Capone et.al. [2], using an extension of DMFT that embodies both orbital degeneracy and local superconducting pairing. They find that close to the Mott transition a superconducting pocket appears, with a greatly enhanced energy gap $\Delta$ as compared to what it would be with $J$ only, without the Coulomb repulsion $U$. As a result the Mott transition is once again hidden, but here by superconductivity instead of antiferromagnetism. The purpose of this note is 
to provide a physical understanding of that important result, which seems to be a generic feature of any Mott transition to an insulator with no residual entropy at $T=0$. Such qualitative arguments were actually presented in the original paper [2]: we modify them somewhat and we hope to clarify the issue.

DMFT considers the limit of an infinite dimension in which all interaction vertices are local. In momentum space they should not depend on momenta. If this were true one could infer the full scattering vertex from its forward scattering limit, which in turn is related to Landau quasiparticle interactions, i.e. to thermodynamic response functions. In principle the Cooper superconducting kernel $K$ should follow. Unfortunately the infinite dimension limit is subtle, as shown in the Appendix: such arguments only yield orders of magnitude. They are nevertheless instructive, as they suggest the sign and order of magnitude of $K$. In section 2 we discuss the usual Hubbard model as a test: we conclude that close to the Mott transition effective quasiparticle interactions are repulsive and of intermediate strength, as expected in a system with only repulsions. Superconductivity can only exist for doped systems and then it is of type $d[3]$. The orbitally degenerate case is discussed in section 3: it confirms the numerical results of [2]. In the conclusion we emphasize the weaknesses of our approach - whose main virtue is simplicity!

\section{Non degenerate regular Hubbard model}

The only interaction is a local repulsion $U$. For a half filled band a Mott transition occurs at a critical $U_{c}$. Using Dynamical Mean Field Theory (DMFT) it was shown [1] that the transition between a paramagnetic conductor and an incoherent paramagnetic insulator (with random local moments and a residual entropy) is first order. The metal and insulator coexist between $U_{c 1}$ (at which the Mott gap $\Delta_{g}$ opens), and $U_{c 2}$ (at which free carriers disappear). Just below $U_{c 2}$ free carriers sit in a narrow resonance peak, with width $\Delta=z D$ much smaller than the bandwidth $D . z$ is the usual renormalization constant that goes to 0 at $U_{c 2}$. Near the transition, free carriers are heavy fermions and one can view the paramagnetic metal as a Kondo alloy with a Kondo temperature $T_{K} \approx \Delta$. These free carriers quench the residual entropy of the insulator on a small temperature range $\approx T_{K}$, hence the large effective mass. They appear if the energy $T_{K}$ (per site) gained in forming Kondo singlets overcomes the kinetic energy cost $z \Delta_{g}$ in bringing $z$ states 
from the Mott sidebands to the middle of a large preformed gap[4]. The existence of a residual entropy is thus a crucial feature of heavy fermion behaviour. In contrast the spin entropy of the insulator could be quenched by antiferromagnetic order. In such a case the metal-insulator transition remains, but it is due partly to correlations (Mott), partly to Bragg reflection (Slater). The controversy is mostly semantics as both effects coexist. What is not semantics is the lack of a narrow resonance at the symmetry breaking transition.

Since the problem only has a repulsion it is unlikely that superconductivity occurs. It is nevertheless useful to produce a simple argument that explains why. Naive perturbation theory would say that the kernel $U G G$ in the Cooper channel is reduced by a factor $z^{2}$, the weight of the quasiparticle peaks in the two $G$ factors. Since the quasiparticle density of states is $\widetilde{\rho}=\rho / z$ the resulting dimensionless quasiparticle interaction $\tilde{\rho} U z^{2}$ should be a weak repulsion of order $z$. Put another way the interaction energy is $U z^{2} \approx \Delta z$. But does perturbation theory make sense? An alternate approach is to use the Landau picture of a quasiparticle Fermi liquid in order to estimate the forward scattering limit of quasiparticle interactions. Sure, forward scattering is not full scattering - but it provides a starting point! We start from the usual thermodynamic response functions, expressed in terms of the quasiparticle interaction $f_{k k^{\prime}}$. We show in the Appendix that only the $\ell=0$ part survives in infinite dimension: the dimensionless $F_{k k^{\prime}}=\widetilde{\rho} f_{k k^{\prime}}$ thus depends on two numbers $F_{o s}$ and $F_{o a}$. The charge compressibility is

$$
\kappa=d n / d \mu=\frac{\widetilde{\rho}}{1+F_{o s}}
$$

$\kappa$ vanishes in the insulator (moving $\mu$ inside the gap $\Delta_{g}$ makes no difference). By continuity $\kappa$ must be $\approx z$ near the transition, as confirmed by the detailed DMFT calculation. It follows that $F_{o s} \approx 1 / z^{2}$. In contrast the global spin suceptibility (with a $g$-factor at every site) is

$$
\chi=d M / d H=\frac{\tilde{\rho}}{1+F_{o a}}
$$

Due to the "Anderson compensation theorem" $\chi$ is an unrenormalized constant $\approx \rho$. Because spin is conserved, the Zeeman splitting can be absorbed in a redefinition of the bare particle energies $\epsilon_{k}$ : the only change is a shift of the band edges in opposite directions for up and down spins. Nothing happens at the Fermi level and consequently $=\chi$ ignores the existence of the 
resonance. It follows that $F_{o a} \approx 1 / z$. We thus know the Landau coefficients, which are both singular as $z$ goes to 0 . Note that within perturbation theory $f_{k k^{\prime}}$ corresponds to the so called " $\omega$-limit", in which momentum transfer goes to 0 first: in that limit the Fermi level singularity does not contribute to the Bethe-Salpeter kernel. It is thus surprising that $f$ should be singular as a function of $z$ ! The answer is that such a singularity is not a quasiparticle effect, but a consequence of the residual entropy of the Mott sidebands. Anyhow, whatever its origin, the calculation of $F_{o s}$ and $F_{o a}$ is reliable.

The Cooper kernel involves large momentum transfers: its forward scattering limit thus corresponds to the so called " $k$-limit", in which momentum transfer goes to 0 last. That forward scattering amplitude is also provided by Landau theory: its dimensionless version is

$$
A_{o s}=\frac{F_{o s}}{1+F_{o s}} \approx 1-\frac{1}{F_{o s}} \quad, \quad A_{o a}==\frac{F_{o a}}{1+F_{o a}} \approx 1-\frac{1}{F_{o s}}
$$

The amplitude for antiparallel spins is consequently

$$
A_{\uparrow \downarrow}=A_{o s}-A_{o a} \approx z
$$

The interaction is repulsive because the spin channel dominates. Note that the interaction energy is $\Gamma_{\uparrow \downarrow}=A_{\uparrow \downarrow} / \widetilde{\rho} \approx D z^{2}$, in agreement with the naive calculation. We conclude that the effective dimensionless interaction is reduced by renormalization, by a factor $z$, but it remains repulsive.

The physics of quasiparticle pairs is controlled by the $\ell=0$ interaction $\gamma$ in the particle-particle channel. If the interaction were local, nothing would depend on momentum and $\gamma$ would be the same as forward scattering in the particle-hole channel. Unfortunately that is not tenable. A purely local interaction implies a zero scattering amplitude $\gamma_{\uparrow \uparrow}$ because of crossing symmetry (exclusion principle), in clear disagreement with the exact calculation of $F$ and $A$. We show in the Appendix that indeed $\gamma$ is non local. Thus we cannot relate $\gamma$ to forward scattering. But a reasonable guess is that sign and order of magnitude are preserved. If so, $s$-wave superconductivity is impossible in the half filled non degenerate Hubbard model.

\section{Orbitally degenerate Hubbard model}

We consider now a model in which one electron on a given site has an orbital angular momentum $L=1$, in addition to the two spin states $S=1 / 2$. A 
large on site Coulomb repulsion $U$ acts between all local channels

$$
\frac{1}{2} U n^{2} \quad, \quad n=\sum_{m, \sigma} n_{m \sigma}=\text { localvalency }
$$

For any integer $n$, from 1 to 5 , a Mott transition should occur when $U$ exceeds a threshold $U_{c}$. Basically the system becomes an insulator when the splitting of Mott sidebands exceeds the bandwidth. The transition is primarily a valency effect and degeneracy plays a minor role in its occurrence. Consider for instance the case $n=2$. A given site has 15 states which must be odd under fermion permutation: spin singlet $S=0$ with= either $L=0$ or $L=2$, spin triplet $S=1$ with $L=1$. All these states are degenerate if only $U$ is there. Then the issue of residual entropy is still around - just worse!

We now add a small interaction $J$ that lifts that degeneracy. We ignore Hund's rule and we assume that the lowest state is the singlet $S=0, L==0$. Of course physical arguments should be supplied to support that - but at this stage we take it as a model. Then the entropy of localized electrons is naturally quenched on a temperature range of order $J$. The ground state has no residual entropy: the issue of translational symmetry breaking (such as antiferromagnetism) is not as pressing as in the usual Hubbard model. Assume that the Mott transition is approached from the metallic side: as long as the resonance width $\Delta$ is larger than the splitting $J$, the latter should be irrelevant! In more physical terms, $\hbar / \Delta$ is the flipping time of the local state, meaning a finite time memory in much the same way as a temperature means a memory $\hbar / T$ : any longer time scale is irrelevant. If $J \ll U, D$, the Mott gap will first develop as if $J$ were 0 . The arguments of the preceding section remain valid and a narrow resonance inside a large preformed gap picture should develop: at zero temperature the resonance width $\Delta$ decreases as $U$ approaches the Mott transition $U_{c 2}$. Such a picture must break down when $\Delta$ reaches $J$. Then local splitting takes over free carriers in quenching the entropy: the very mechanism that led to free carriers (the gain in Kondo energy pays for the cost of kinetic energy) does not operate any more. Some new physics should take over!

Note that $J$ is an atomic entity, which does not care much about mobile carriers: it should be insensitive to renormalization (as we will show later). In contrast we expect $U$ to scale down to 0 as one approaches the Mott transition, just as for the regular Hubbard model. In order to understand that difference, we note that the decrease of $U$ expresses the quenching of 
charge fluctuations due to localization, while $J$ characterizes the level structure within a given valency, in a manifold where charge fluctuations are irrelevant. Assume for a moment that it is true. Then the interaction $J$ that pushes the singlet down acts like an attraction between quasiparticles: is it possible that such an attraction creates superconductivity? Then a spectacular strong coupling regime should emerge close to $U_{c 2}$. We try to answer that question within the qualitative approach used in the preceding section.

Let us assume that $\Delta \approx J$ : the resonance is not much affected by $J$, the density of states is $\widetilde{\rho}=\rho / z \approx \rho D / \Delta$. Can we estimate the Cooper vertex from thermodynamic response functions, using Landau theory for the forward scattering amplitude? Since we are dealing with singlet pairs, the convenient basis for treating spin 1 particles is the cartesian basis $x, y, z$, rather than angular momentum $m=-1,0,+1$. A singlet pair is the sum

$$
(x \uparrow, x \downarrow)+(y \uparrow, y \downarrow)+(z \uparrow, z \downarrow)
$$

(which is clearly rotation invariant). The corresponding Cooper kernel is $=M+2 N$, where $M$ scatters $(x \uparrow, x \downarrow)$ into itself and $N$ scatters $(x \uparrow, x \downarrow)$ into $(y \uparrow, y \downarrow)$. We must transfer that signature into the particle hole channel in which the Landau parameter $f_{k k^{\prime}}$ acts. For $M$ that transfer leads to

$$
(\mathrm{el} x \uparrow, \text { ho } x \uparrow) \Longrightarrow(\mathrm{el} x \downarrow, \text { ho } x \downarrow)
$$

Focussing on the orbital part we write

$$
c_{x}^{*} c_{x}=\frac{1}{3}\left[\left(c_{x}^{*} c_{x}+c_{y}^{*} c_{y}+c_{z}^{*} c_{z}\right)+\left(c_{x}^{*} c_{x}-c_{y}^{*} c_{y}\right)+\left(c_{x}^{*} c_{x}-c_{z}^{*} c_{z}\right)\right]
$$

We can infer $M$ from a combination of spin symmetric and antisymmetric response to charge and quadrupolar perturbations. The calculation of $N$ involves the particle hole vertex

$$
(\mathrm{el} x \uparrow, \text { ho } y \uparrow) \Longrightarrow(\mathrm{el} y \downarrow \text {, ho } x \downarrow)
$$

If a rotation by $\pi / 4$ is applied, the quadrupolar vertex $\left(c_{x}^{*} c_{x}-c_{y}^{*} c_{y}\right)$ transforms into $\left(c_{x}^{*} c_{y}+c_{y}^{*} c_{x}\right) . N$ is a purely quadrupolar quantity. Let $\Gamma$ be the scattering vertex in the particle-hole channel. The forward scattering limit may be written as

$$
|\alpha|^{2}\left(A_{\rho s}-A_{\rho a}\right)+|\beta|^{2}\left(A_{Q s}-A_{Q a}\right)
$$


where $\alpha$ and $\beta$ are angular coefficients, $A_{\rho}$ and $A_{Q}$ interactions in the charge and quadrupolar channels, which can be inferred from the corresponding thermodyanmic response functions.

The $\Gamma_{\rho}$ part involves the compressibility and the spin susceptibility: it yields a small repulsion as in the regular Hubbard model, with a dimensionless $\widetilde{\rho} \Gamma$ of order $z$. The interesting part is the quadrupolar $\Gamma_{Q}$ which involves virtual excitation to excited atomic states within the manifold of valency 2 . Within Fermi liquid theory let us write

$$
\chi_{Q s}=\frac{\widetilde{\rho}}{1+F_{Q s}} \quad, \quad \chi_{Q a}=\frac{\widetilde{\rho}}{1+F_{Q a}}
$$

(which fixes the normalization of the quadrupole). The dimensionless forward scattering amplitudes are $A_{i}=F_{i} /\left(1+F_{i}\right)$, leading to

$$
A_{Q s}-A_{Q a}=\frac{F_{Q s}}{1+F_{Q s}}-\frac{F_{Q a}}{1+F_{Q a}}=\frac{1}{1+F_{Q a}}-\frac{1}{1+F_{Q s}}=\frac{\chi_{Q a}-\chi_{Q s}}{\tilde{\rho}}
$$

The whole issue is an estimate of $\chi_{Q s}$ and $\chi_{Q a}$.

The Anderson compensation theorem should not apply since the conservation of $L$ is not as strict as that of $S$, the less so if crystal field perturbations lift the orbital rotational invariance. Let us first consider the insulator: the quadrupolar response functions are primarily an atomic problem and we expect them to be of order $1 / J$, independent of the resonance width $z D$ in lowest order. More specifically $\Gamma_{Q s}$ involves an external field which is an orbital quadruplet and a spin singlet. It naturally couples the singlet ground state $S=L=0$ into the excited configuration $S=0, L=2$ : we thus expect $\chi_{Q s} \approx \rho D / J$, where $\rho$ is the bare density of states. In contrast $\Gamma_{Q a}=$ involves an external field which is an orbital quadruplet and a spin triplet: it should couple the ground state to an $S=1, L=2$ state which does not exist. Any response must involve virtual coupling to neighbouring sites, similar to the picture of van Vleck paramagnetism. At best we expect a small $\chi_{Q a}$ of order $\rho$. Note that we thereby automatically generate an attraction, which is hardly surprising since we assumed that the singlet was lowest!

Such a result only holds if $\Delta<J$ (the bandwidth should not compete with local splitting). The dimensionless Cooper vertex (scaled by the density of states $\tilde{\rho}$ ) is then

$$
A_{Q s}-A_{Q a} \approx-\frac{\Delta}{J}
$$


We see that in the crossover region $\Delta \approx J$ the effective quasiparticle interaction is an intermediate strength attraction! The real attraction energy is comparable to the bandwidth $\Delta$. In the opposite limit $\Delta \gg J$ the Hund coupling $J$ becomes negligible: the susceptibilities $\chi_{Q s}$ and $\chi_{Q a}$ should be controlled by the free particles and $J$ can be set equal to 0 . Both $\chi_{Q s}$ and $\chi_{Q a}$ should be equal to $\widetilde{\rho}$ and $A_{Q s}-A_{Q a}$ should consequently vanish.

We thus confirm our initial guess: the "Hund" coupling between quasiparticles, originally $J$, is essentially unchanged until the bandwidth is comparable to $J$, precisely when the entropy quenching changes regime, from a Kondo picture to a more conventional atomic mechanism. This is contrasted to the effective $U$ which is renormalized to 0 as the Mott transition is approached. In the crossover region the effective quasiparticle interaction is an intermediate strength attraction, which goes down to 0 when $\Delta \ll J$, disappearing at the Mott transition.

Up to now the discussion is only concerned with forward scattering. Just as for the regular Hubbard model we cannot proceed to the real Cooper kernel for superconductivity. But, once again, we can reasonably assume that a partly non local interaction should not change orders of magnitude and signs. If this is so, a pocket of s-wave superconductivity should exist close to the Mott transition, which is exactly the result of [2]. The control parameter is the ratio $\Delta / J$. When $\Delta \gg J$ the Coulomb repulsion dominates because it has not scaled down far enough. Superconductivity appears in the crossover region, first in a strong coupling regime analogous to Bose Einstein condensation or preformed pairs. When $\Delta$ becomes $\ll J$, superconductivity switches to a BCS regime, ultimately disappearing at the Mott transition "faute de combattants".

Such a qualitative argument looks robust. It teaches us a number of interesting points:

(i) Some sort of local attraction must exist in order to produce s-wave superconductivity. Here it is the Hund coupling $J$.

(ii) Superconductivity competes with translational symmetry breaking in quenching the high temperature entropy. It seems that a singlet ground state is crucial in eliminating the appearance of two sublattices. In this respect the simpler choice of an orbital "flavour" doublet would not work. The antisymmetric two particle states are $S=0, L=1$ or $S=1=, L=0$ : none of them is a singlet!

(iii) The energy scale that emerges is the unrenormalized $J$. As long as $J$ is smaller than the Mott gap $\Delta_{g}$ the description in terms of a narrow resonance 
holds. If $J$ is larger one moves into genuine strong coupling superconductivity, which is another story.

(iv) The Fermi liquid crosses over from a weak coupling regime at small $U$ to a strong coupling regime near the Mott transition. In order to generate an attraction in the Cooper singlet channel the spin symmetric quadrupolar susceptibility must be larger than its spin antisymmetric counterpart - a condition fulfilled by a pair of $L=1$ electrons.

These remarks may provide hints in searching for new materials.

\section{Conclusion}

The above qualitative arguments do not add much to the beautiful results of [2]. The main point is the statement that a superconducting pocket should automatically appear near a Mott transition towards an insulator whose ground state is a singlet, with an unconventional strong coupling behaviour in the crossover region. Because that statement is qualitative, it may help searching for specific examples. Playing with an inverted Hund's coupling looks dangerous: exchange is a robust feature of atomic physics! Materials scientists versed in the infinite variety of transition metals and rare earths compounds may find good examples. I want here to draw attention to another possibility, namely molecular crystals. There are many molecules whose ground state is a singlet - say ethylene with a pair of bonding $\pi$ electrons. One can certainly find molecules where the singlet excitation energy is small. If they are put under pressure they might undergo a metal-insulator transition: do they display strong coupling superconductivity near the transition? The answer is not obvious, for a number of reasons. There are many alternatives to a metal-insulator transition - for instance polymerization: this is certainly what happens to compressed ethylene. One may try to stop it grafting methyl groups on the double bond - but then the coherent band width of the metallic phase is dramatically reduced. Another problem is the possibility of a first order transition, due for instance to lattice distortions, which will hide the critical region where $\Delta$ goes to 0 . On more theoretical grounds one should not forget that the sign of the interaction depends on the nature of molecular

excited states (remember our argument which was based on the absence of an $L=2, S=1$ atomic state!). All these warnings show that the quest is by no means obvious! But nature is rich: the main purpose of this short note was to launch the question and to provide a few guidelines. An experimental 
(a)

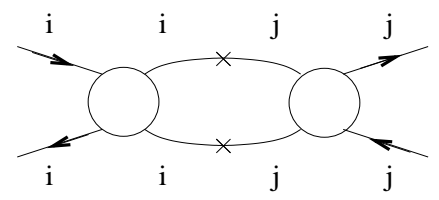

(b)

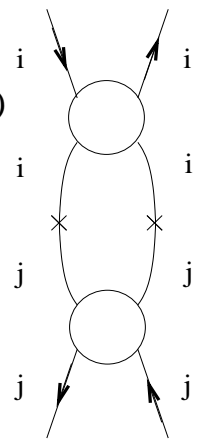

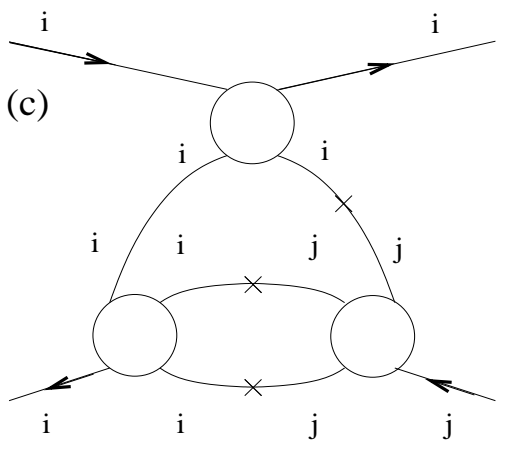

Figure 1: A few example of non local vertices (the crosses are hopping matrix elements $t$ ). (a) Hopping of a local pair $(i, i)$ to $(j, j)$. (b) Onsite interaction within a local pair. (c) Breaking of an incoming local pair.

realization of the proposal of [2] would enhance the impact of a really original result!

Acknowledgements: the author wishes to thank Michele Fabrizio for communicating his manuscript prior to publication, and for many stimulating discussions. This note dwells on his ideas.

\section{A Appendix}

In this appendix we try to clarify the nature of interaction vertices in infinite dimension $d$. Let $t$ be the hopping amplitude to a nearest neighbour site: due to destructive interference the effective bandwidth is $D \approx t \sqrt{d}$ with negligible tails until $t d$. The large coordinence tends to make the vertices local. The standard example is the one particle self energy $\Sigma_{i j}(\varepsilon)$. Any intermediate state has at least three lines. Since the interaction is local, entering at $i$ and exiting at $j$ implies non local propagators $G_{i j}$ inside the self energy. The minimum number of these is 3 : assuming intermediate coupling $U \approx D$, a non local element $i \neq j$ is of order $t^{3} / D^{2} \approx t / d$, negligible as compared to the bare hopping $t$. When expressed in terms of skeleton graphs, $\Sigma_{i i}\left[G_{i i}\right]$ is consequently a local functional of the full $G_{i i}$. Site $i$ knows the outside world only through $G_{i i}$, via excursions to nearby sites, equivalent to an additional local self energy

$$
\sigma=\sum_{j} t^{2} G_{j j}
$$


Excursions involve a single line and the summation over $j$ makes for the factor $1 / d$ in $t^{2}$.

We want to carry that discussion to a scattering amplitude described by a four point vertex, which can be read either in the ppchannel $(\gamma)$ or in one of the two $p h$ channel $(\Gamma)$. We work in real space instead of momentum space. Disregarding for a moment energy and spin variables, the vertex in a given channel is indexed by the entering sites $i_{1} i_{2}$ and by the exit sites $j_{1} j_{2}$. The local term with all sites equal will clearly dominate as it does for the self energy: to what extent should we worry with non local terms? Let us start from $\Gamma_{i i}^{i i}$ and go step by step. The simplest case is the hop of a local pair to another local pair, $\Gamma_{i i}^{j j}$. If the diagram is irreducible in the channel under consideration (it cannot be split by cutting 2 lines only), then the minimum number of intermediate lines is 4 : the corresponding irreducible interaction $I_{i i}^{j j}$ is of order $t^{4} \approx 1 / d^{2}$, thus negligible. If on the other hand the diagram is reducible (Fig.1a), there exists an intermediate state with 2 lines: if the hopping only acts there the contribution is of order $t^{2} \approx 1 / d$. When writing the Bethe-Salpeter equation

$$
\Gamma_{i i}^{j j}=I \delta_{i j}+I G_{i n} G_{i n} \Gamma_{n n}^{j j}
$$

we see that non local vertices $\Gamma$ are of order $1 / d$, but they are relevant once one sums over $n$ and $j$. A similar argument holds for $\Gamma_{i j}^{i j}$, which scatters particles sitting on neighbouring sites. A diagram which is reducible in the cross channel, as shown on Fig1b, yields a contribution $\approx 1 / d$ which is relevant upon summation over $j$. This is not so for terms such as $\Gamma_{i i}^{i j}$ which would break a local pair. The lowest order diagram shown on Fig.1c yields a contribution of order $t^{3}$. Iteration of these non local pairs reduces the vertex more and more. In physical terms the probability that particles starting from different sites meet in the outside world is negligible.

Let us focus on the particle-hole channel. Moving to momentum space we may write the vertex as

$\Gamma\left(k, k^{\prime} ; q\right)=\sum_{r_{i}} \Gamma\left(r_{i}\right) \exp i\left[k\left(r_{1}-r_{2}\right)-k^{\prime}\left(r_{3}-r_{4}\right)+\frac{q}{2}\left(r_{1}+r_{2}-r_{3}-r_{4}\right)\right]$

The fully local $\Gamma_{i i}^{i i}\left(\omega_{i}\right)$ is the dominant term, momentum independent. If we look at it in the $p p$ channel, $\Gamma$ splits into a triplet and a singlet term. Crossing symmetry implies a zero triplet part on the energy shell, $\omega_{i}=\mu$ (antisymmetry upon permutation). Carried over to the usual description in 
terms of spin symmetric and antisymmetric parts $\Gamma^{(s)}$ and $\Gamma^{(a)}$ in the particlehole channel, that means $\Gamma^{(s)}=-\Gamma^{(a)}$ (it is just what happens in first order in the Hubbard $U$ ). In higher orders such a result is clearly inconsistent with Fermi liquid theory, which predicts different limits of the vertex $\Gamma$ in the so called $q$ and $\omega$ limits. The scattering vertex has to be non local: put another way, fermions must move in order to produce a liquid! In practice Landau coefficients such as $f_{k k^{\prime}}$ imply an average of $k$ and $k^{\prime}$ over the Fermi surface, leading to strong destructive interference if $r_{1} \neq r_{2}$ or $r_{3} \neq r_{4}$. It follows that the entering and exiting pairs are local: $\Gamma$ does not depend on $k$ and $k^{\prime}$ (as stated in the paper, the Landau coefficients only have $\ell=0$ terms). In contrast Landau coefficients involves $q \equiv 0$ : one does sum over $\left(r_{1}-r_{3}\right)$ ! That relaxes the crossing symmetry constraint, while precluding any exact relation between forward scattering and Cooper kernel.

\section{References}

[1] A. Georges, G. Kotliar, W. Krauth, M. Rozenberg, Rev. Mod. Phys. 68, $13(1996)$

[2] M. Capone, M. Fabrizio, C. Castellani, E. Tosatti, Science, 296, 4 $(28 / 6 / 2002)$

[3] See for instance A. Paramekanti, M. Randeria, N. Trivedi, Phys. Rev. Lett. 87, 217002 (2001)

[4] Ph. Nozières, Eur. Phys. J. B6, 447 (1998) 